\title{
Crystallographic Microstructure Study of a Japanese Sword made by Noritsuna in the Muromachi Period by Pulsed Neutron Bragg-Edge Transmission Imaging
}

\author{
Hirotaka Sato ${ }^{1, a^{k}}$, Yoshiaki Kiyanagi ${ }^{2, b}$, Kenichi Oikawa ${ }^{3, c}$, Kazuma Ohmae $^{2, \mathrm{~d}}$, \\ Anh Hoang Pham ${ }^{4, e}$, Kenichi Watanabe ${ }^{2, f}$, Yoshihiro Matsumoto ${ }^{5, g}$, \\ Takenao Shinohara ${ }^{3, \mathrm{~h}}$, Tetsuya Kai $\mathrm{i}^{3, \mathrm{i}}$, Stefanus Harjo ${ }^{3, \mathrm{j}}$, Masato Ohnuma ${ }^{1, \mathrm{k}}$, \\ Shigekazu Morito ${ }^{4,1}$, Takuya Ohba ${ }^{4, m}$, Akira Uritani $^{2, n}$ and Masakazu Itoh ${ }^{6,0}$ \\ ${ }^{1}$ Faculty of Engineering, Hokkaido University, Hokkaido 060-8628, Japan \\ ${ }^{2}$ Graduate School of Engineering, Nagoya University, Aichi 464-8603, Japan \\ ${ }^{3}$ J-PARC Center, Japan Atomic Energy Agency (JAEA), Ibaraki 319-1195, Japan \\ ${ }^{4}$ Interdisciplinary Faculty of Sci. and Engineering, Shimane University, Shimane 690-8504 \\ Japan \\ ${ }^{5}$ Comprehensive Research Organization for Science and Society, Ibaraki 319-1106, Japan \\ ${ }^{6}$ Wakou Museum, Shimane 692-0011, Japan

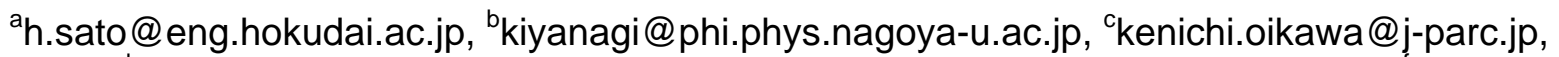 \\ doomae.kazuma@f.mbox.nagoya-u.ac.jp, eanhpham@riko.shimane-u.ac.jp, "k- \\ watanabe@energy.nagoya-u.ac.jp, 9y_matsumoto@cross.or.jp, 'takenao.shinohara@j-parc.jp, \\ itetsuya.kai@j-parc.jp, jstefanus.harjo@j-parc.jp, kohnuma.masato@eng.hokudai.ac.jp, \\ Imosh@riko.shimane-u.ac.jp, mohba@riko.shimane-u.ac.jp, nuritani@energy.nagoya-u.ac.jp, \\ ${ }^{\circ}$ masakazu_itoh@cup.ocn.ne.jp
}

Keywords: Japanese Sword, Noritsuna, Bizen, Muromachi Period, Martensite Phase, Crystallographic Texture, Microstructure, Crystalline Grain, Neutron Imaging, Bragg Edge

Abstract. Large-area real-space distribution of crystallographic microstructural information in a Japanese sword made by Noritsuna at Bizen in A.D. 1405 was non-destructively investigated by Bragg-edge neutron transmission imaging using the RADEN instrument at BL22 of MLF (Materials and Life Science Experimental Facility) in J-PARC and the data analysis software RITS (Rietveld Imaging of Transmission Spectra), as one of the series of a systematic research project. As a result, unique properties of the Noritsuna sword were revealed as follows. Hard martensite which $d$-spacing is close to that of a modern quenched steel exists at the cutting edge, but the area is smaller than that of a modern sword. Coarse grains exist near the notch at the back of the tang. Fine and coarse crystallite-size steels are separately distributed. The texture is not so strong, and the preferred orientation $<210>$ is perpendicular to the normal direction of the sword plate except for the front region of the tang region.

\section{Introduction}

Japanese sword is one of the most interesting cultural-heritage artifacts. In fact, a sword typically consists of multiple-phase structures (e.g., ferrite, cementite and martensite), it was crafted in many steps (e.g., selective quenching (hardening) and iterative hammering etc.), and the manufacturing process varied depending on the era and the geographical location. However, being a tradition orally transmitted, there are many unknown information due to lack of written records, in particular, about ancient swords. Therefore, systematic metallurgical characterizations 
are indispensable for investigating the history of Japanese swords. For this reason, a systematic research project was launched by the authors of this paper. This project aims to non-destructively characterize Japanese swords made by swordsmiths in various places and eras, by using neutron diffraction (S. Harjo, K. Oikawa et al.), neutron tomography (Y. Matsumoto, K. Watanabe et al. [1]) and Bragg-edge neutron transmission imaging. From viewpoints of both metallurgical characterization inside a bulk and valuable art/heritage preservation, we consider the best method is a neutron beam experiment like previous works performed by neutron diffraction [2], tomography [3] and Bragg-edge transmission imaging [4,5].

In cooperation with Wakou Museum, we systematically investigated four Japanese swords by Bragg-edge neutron transmission imaging; a sword made by Morikage at Bizen in 3rd quarter of 14th century (by A. H. Pham et al.), a sword made by Noritsuna at Bizen in 1st quarter of 15th century (this work), a sword made by Sukemasa at Izumi in 1st quarter of 16th century (by K. Oikawa et al. [6]) and a modern sword made by Masamitsu in A.D. 1969 (by K. Ohmae et al. [7]). This paper reports characterization results of one of the series, the Noritsuna sword.

\section{Specimen: Japanese sword made by Noritsuna at Bizen in A.D. 1405}

The Japanese sword investigated in this work was made by swordsmith Noritsuna at Bizen in the Muromachi period (A.D. 1336-1573). There were five famous traditional sword-making styles (schools) “Gokaden”, i.e., Soshu, Mino, Yamashiro, Yamato and Bizen, in the old-sword (Koto) age in A.D. 987-1596. The investigated sword was made at one of the Gokaden, Bizen.

Three regions in the sword, the tip region (Tip), the tang region (Tang) and the region between Tip and Tang (Middle), were measured by wavelength-resolved neutron radiography.

\section{Pulsed neutron imaging experiment and Bragg-edge transmission spectrum analysis method}

Time-of-flight (TOF) neutron radiography experiment was performed at the RADEN instrument [8] at BL22 of MLF (Materials and Life Science Experimental Facility) in J-PARC which was the energy-resolved neutron imaging instrument connected to a megawatt-class pulsed spallation neutron source. The power of the $3 \mathrm{GeV}$ proton synchrotron was $150 \mathrm{~kW}$ during this experiment. The neutron moderator was a decoupled-type supercritical para- $\mathrm{H}_{2}$ moderator. The sword and a detector were placed at $24 \mathrm{~m}$ from the neutron source. The collimator ratio L/D was 1000 since a pinhole placed at $8 \mathrm{~m}$ from the neutron source was set in the diameter of $15 \mathrm{~mm}$. The wavelength resolution $\Delta \lambda / \lambda$ at $\lambda=0.4 \mathrm{~nm}$ was $0.2 \%$. The used neutron TOF-imaging detector, nGEM, was a GEM (gas electron multiplier) type detector [9] which had the pixel size of $800 \mu \mathrm{m}$ and the detection area of $10.24 \mathrm{~cm} \times 10.24 \mathrm{~cm}$. The measurement time were 7.1 hours for Tip, 8.4 hours for Middle, 8.2 hours for Tang and 6.0 hours for open beam. The measurement time was relatively long due to limit of the maximum counting rate of the detector.

The used data analysis software was the latest version of RITS (Rietveld Imaging of Transmission Spectra) [10-12] for analysis of Bragg-edge neutron transmission spectra at each pixel. Since Bragg-edges in wavelength-dependent neutron transmission data (spectrum) are caused by diffraction phenomenon of neutrons, we can get crystallographic microstructural information of a specimen by analyzing the Bragg-edge transmission spectrum. The single Bragg-edge wavelength-position/broadening analysis mode of RITS [11], which we can exploit to obtain the information of the average value and the full width at half maximum (FWHM) of distribution of crystal lattice plane spacing ( $d$-spacing), was used for mapping martensite phase in steel, similarly to a previous work [11]. On the other hand, the Rietveld-type (wide wavelength bandwidth) Bragg-edge transmission spectrum analysis mode of RITS [10] was used for evaluating the information of projected atomic number density (effective thickness), preferred orientation (the most probable crystal orientation) along the beam direction, degree of 
crystallographic texture (microstructure with anisotropic crystal orientation distribution) evolution and crystallite size. The initial Bragg-edge broadening profile due to only the instrumental resolution was determined by measuring a standard $\alpha$-iron (ferrite).

\section{Results of single Bragg-edge analysis for imaging of martensite phase in the sword}

Figs. 1 (a) and (b) show two types of spectrum near $\{110\}$ Bragg-edge of $\alpha$-iron and the profile fitting results obtained by the single Bragg-edge analysis mode of RITS [11], at the blade body region (Position A, see Fig. 2) and the cutting-edge region (Position B, see Fig. 2). It was confirmed that Bragg-edge of the cutting-edge region was broadened due to presence of martensite. In fact, it is well known that the cutting edge is hardened by quenching resulting in the formation of a martensite structure. The measured Bragg-edge broadening reflected it, and this was consistent with a previous work [5].
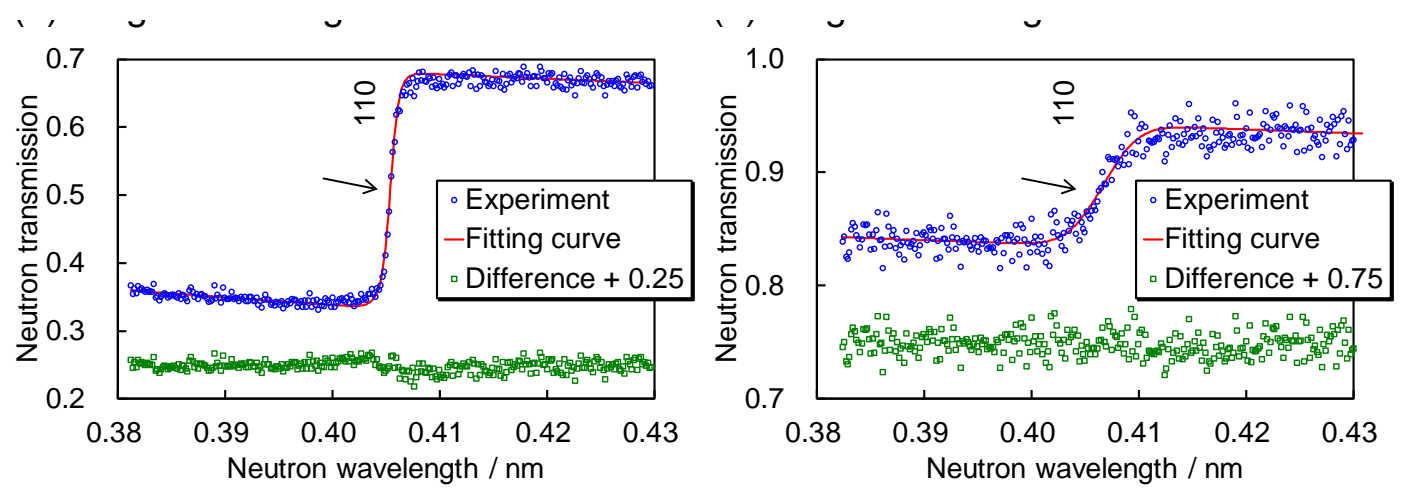

Fig. 1: Results of single Bragg-edge (BE) fitting analysis at (a) the blade body region and (b) the cutting-edge region.

(a) $d_{110}$ at Tang

Average value of distribution of crystal lattice plane spacing, $d_{110}^{\prime}(\mathrm{nm})$

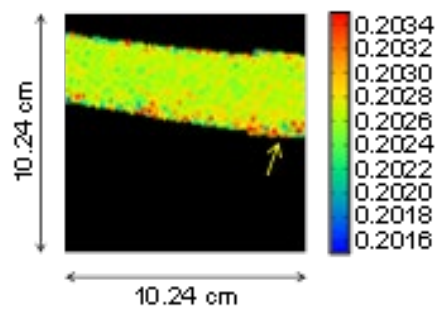

(d) $w_{110}$ at Tang

F'vi'HM of distribution of

crystal lattice plane spacing, $w_{110}(\mathrm{~nm})$

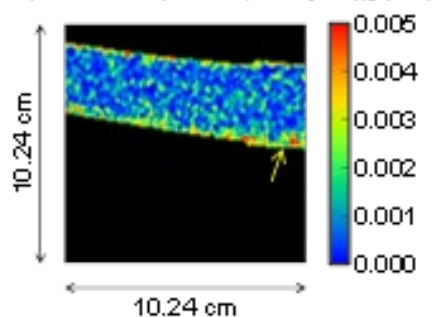

(b) $d_{110}$ at Middle

Average value of distribution of crystal lattice plane spacing, $\mathrm{d}_{110}(\mathrm{~nm})$

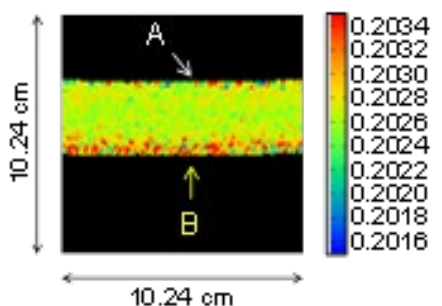

(e) $w_{110}$ at Middle

F'o' $\mathrm{HM}$ of distribution of

crystal lattice plane spacing, $w_{110}(\mathrm{~nm})$

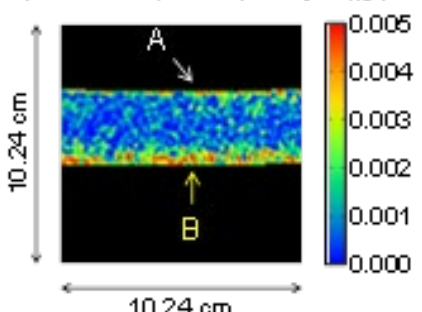

(c) $d_{110}$ at Tip

Awerage value of distribution of crystal lattice plane spacing. $d_{110}^{\prime}(\mathrm{nm})$

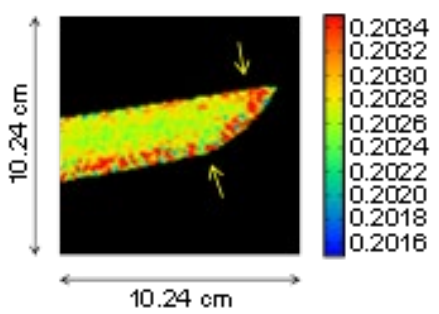

(f) $w_{110}$ at Tip

F'DiH Mist of distribution of crystal lattice plane spacing, $w_{110}(\mathrm{~nm})$

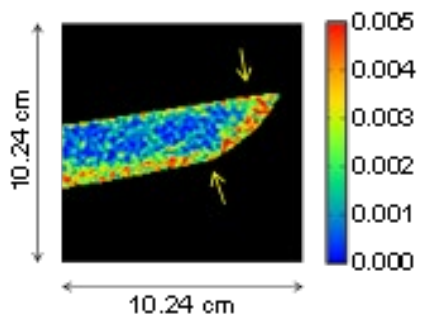

Fig. 2: Imaging results of average value $\left(d_{110}\right)$ and FWHM $\left(w_{110}\right)$ of the distribution of $\{110\}$ crystal lattice plane spacing at Tang, Middle and Tip, obtained by single Bragg-edge profile fitting analysis. 
Figs. 2 (a)-(c) show maps of the average value of $d$-spacing distribution $\left(d_{110}\right)$, and Figs. 2 (d)-(f) show results of FWHM of $d$ spacing distribution $\left(w_{110}\right)$, at each region (Tang, Middle and Tip). High $d_{110}$ and $w_{110}$ indicate existence of martensite phase in steel, and low $d_{110}$ and $w_{110}$ indicate existence of ferrite phase in steel [11]. The absolute values of $d_{110}$ and $w_{110}$ evaluated in this sword are close to those evaluated in a previous work [11]. Namely, the imaging results show that martensite phase (quenched and hardened region) exists near the cutting edge (yellow arrows in Fig. 2). In addition, an interesting aspect of the Noritsuna sword is the width

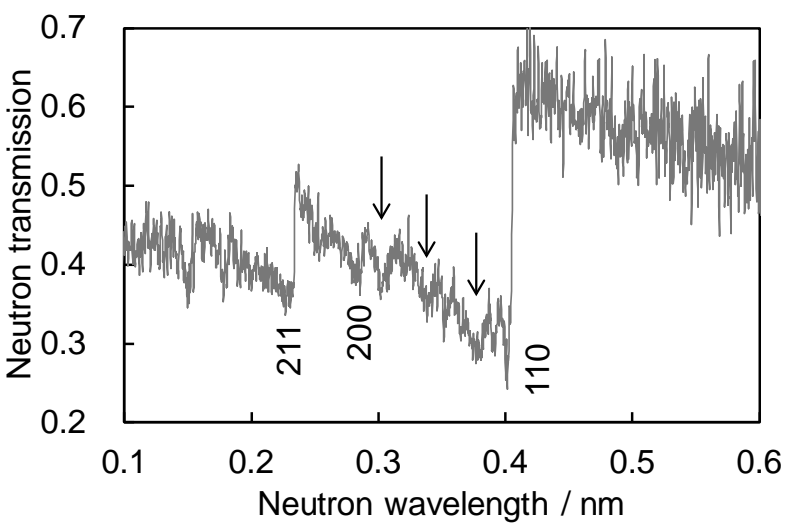

Fig. 3: Coarse-grain type transmission spectrum observed near notch at the back of Tang (Position C). (area) of the martensite region. The width was smaller than that of a modern sword [7]. This means two possibilities; modern sword-quenching process was more well-controlled than ancient one because of the advance in metallurgical knowledge, or the hardened cutting-edge was lost by re-sharpening of the sword during its service. In any case, although the martensite width is small, the hardness seems not to be low because both $d_{110}$ and $w_{110}$ are close to those of a modern quenched 0.45 mass\%C-steel [11].

Results of Rietveld-type analysis for imaging of microstructural information in the sword First of all, we discover a relatively coarse-grained region in the Noritsuna sword. Fig. 3 shows a
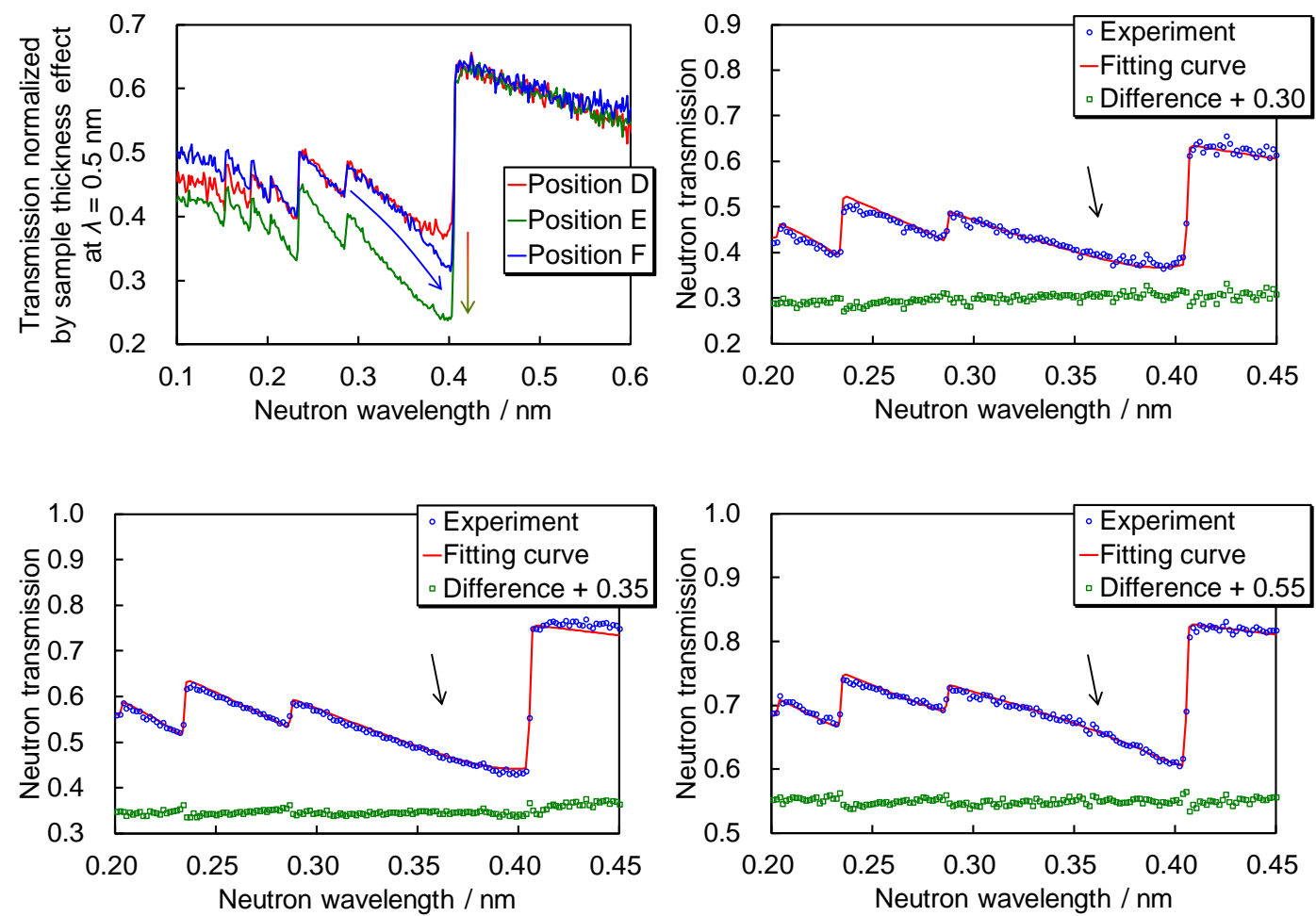

Fig. 4: (a) Change of neutron transmission spectra among Positions D, E and F. Results of Rietveld-type profile fitting analysis for transmission spectra measured at Positions $D(b), E(c)$ and $F(d)$. 
(a) Tang

Projected atomic number density (Thickness) $\left(10^{22}\right.$ atoms $\left./ \mathrm{cm}^{2}\right)$

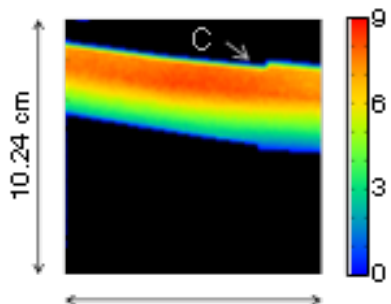

$10.24 \mathrm{~cm}$

(d) Tang

Crystallite s ize $(\mu m)$

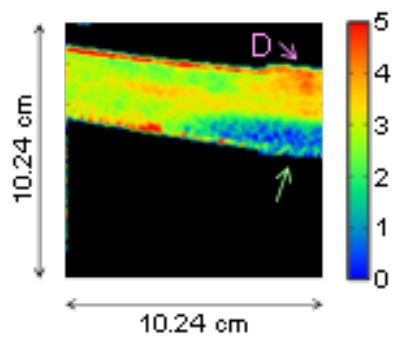

(g) Tang

Degree of crystallographic anis otropy (March-Dollase coefficient)

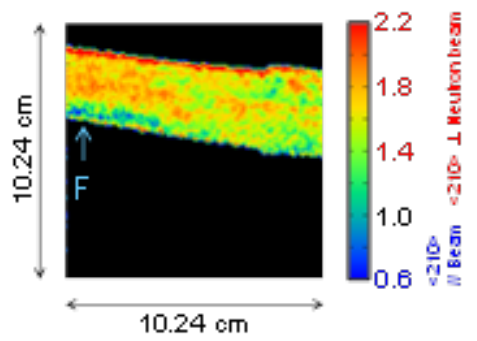

(b) Middle

Projected atomic number density (Thickness) (1022 atoms $/ \mathrm{cm}^{2}$ )

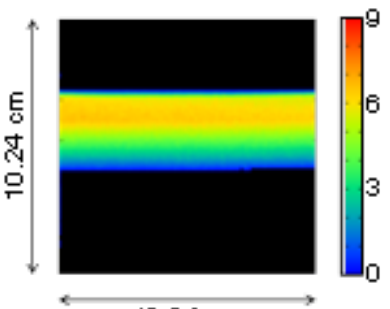

$10.24 \mathrm{~cm}$

(e) Middle

Crystallite s ize $(\mu m)$

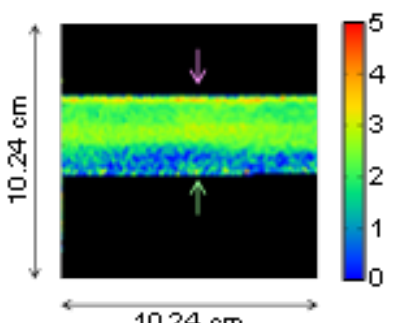

(h) Middle

Degree of crystallographic anis otropy (March-Dollase coefficient)

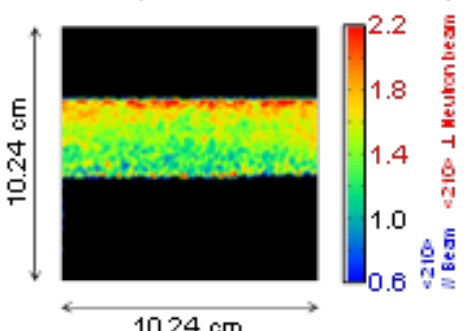

$10.24 \mathrm{~cm}$ (c) Tip

Projected atomic number density (Thickness) $\left(10^{22}\right.$ atome $\left./ \mathrm{cm}^{2}\right)$

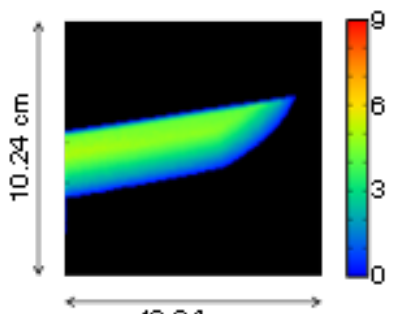

$10.24 \mathrm{~cm}$

(f) Tip

Crystallite size $(\mu m)$

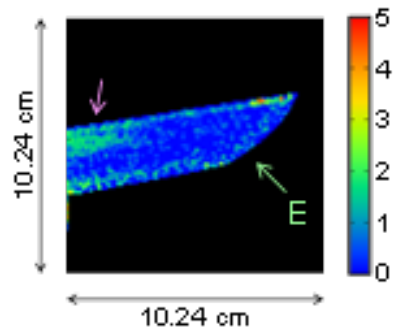

(i) Tip

Degree of cnystallographic anisotropy (March-D ollase coefficient)

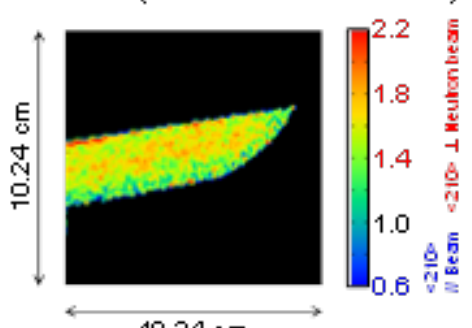

$10.24 \mathrm{~cm}$

Fig. 5: Imaging of projected atomic number density, crystallite size and degree of crystallographic texture evolution.

neutron transmission spectrum measured near the notch at the back of Tang (Position C, see Fig. 5 (a)). Some transmission dips due to relatively-coarse grains [13] were observed here.

Fig. 4 shows neutron transmission spectra with the fitting curves obtained by RITS, at Positions D, E and F (see Fig. 5). Fig. 4 indicates that intensity or shape of transmission spectrum are changed by crystallite size or preferred orientation [10]. The details are discussed as follows.

Fig. 5 shows imaging results of (a)-(c) projected atomic number density, (d)-(f) crystallite size and (g)-(i) degree of crystallographic texture evolution (the March-Dollase coefficient) at Tang, Middle and Tip, obtained by RITS; the detailed information of each parameter was explained in Ref. [10]. From imaging results of crystallite size, it was found that this sword consisted of two types of iron; one was fine-microstructure steel which composed the cutting-edge side and the tip region (green arrows in Figs. 5 (d)-(f)), and the other was coarse-microstructure steel which composed the inner body and the tang region (pink arrows in Figs. 5 (d)-(f)). 
We also found that the main preferred orientation over the whole region in the sword was $<210>$ which was perpendicular to neutron transmission direction (ND), through the Bragg-edge texture analysis using the March-Dollase function of RITS [10]. However, the preferred orientation $<210>$ becomes to be parallel to ND near the front of Tang (Position F, see Fig. 5 (g)). In any case, degree of texture of this sword was generally weak (see Figs. 4 and 5 (g)-(i)).

\section{Conclusion}

We found some interesting information of crystallographic microstructure in a Japanese sword made by Noritsuna, by using Bragg-edge neutron transmission imaging.

(1) The cutting edge consists of hard quenched steel (martensite phase). The width is smaller than that of a modern sword [7], but the hardness seems not to be low because the $d$-spacing distribution of martensite in this sword is close to that of a modern quenched steel [11].

(2) This sword includes coarse grains only near the notch at the back of the tang region.

(3) Both fine-crystallites steel which mainly composes the tip region and coarse-crystallites steel which mainly composes the tang region are separately distributed.

(4) The texture is not so strong. The preferred orientation $<210>$ is perpendicular to ND over the whole region, but becomes to be parallel to ND only around the front of the tang region.

\section{Acknowledgements}

The neutron experiment at J-PARC MLF BL22 "RADEN" was performed under a user program (Proposal No. 2017A0099). This work partially includes the results of "Collaborative Important Researches” organized by JAEA, QST and The University of Tokyo.

\section{References}

[1] Y. Matsumoto, K. Watanabe et al., Mater. Res. Proc. (these proceedings).

[2] F. Grazzi, L. Bartoli, F. Civita, R. Franci, A. Paradowska, A. Scherillo and M. Zoppi, J. Anal. At. Spectrom. 26 (2011) 1030-1039. https://doi.org/10.1039/c0ja00238k

[3] F. Salvemini, F. Grazzi, S. Peetermans, F. Civita, R. Franci, S. Hartmann, E. Lehmann and M. Zoppi, J. Anal. At. Spectrom. 27 (2012) 1494-1501. https://doi.org/10.1039/c2ja30035d

[4] K. Kino, N. Ayukawa, Y. Kiyanagi, T. Uchida, S. Uno, F. Grazzi and A. Scherillo, Phys. Procedia 43 (2013) 360-364. https://doi.org/10.1016/j.phpro.2013.03.043

[5] Y. Shiota, H. Hasemi and Y. Kiyanagi, Phys. Procedia 88 (2017) 128-133.

https://doi.org/10.1016/j.phpro.2017.06.017

[6] K. Oikawa et al., Mater. Res. Proc. (this proceedings).

[7] K. Ohmae et al., Mater. Res. Proc. (this proceedings).

[8] T. Shinohara, T. Kai, K. Oikawa, M. Segawa, M. Harada, T. Nakatani, M. Ooi, K. Aizawa, H. Sato, T. Kamiyama, H. Yokota, T. Sera, K. Mochiki and Y. Kiyanagi, J. Phys. Conf. Ser. 746 (2016) 012007. https://doi.org/10.1088/1742-6596/746/1/012007

[9] S. Uno, T. Uchida, M. Sekimoto, T. Murakami, K. Miyama, M. Shoji, E. Nakano, T. Koike, K. Morita, H. Satoh, T. Kamiyama and Y. Kiyanagi, Phys. Procedia 26 (2012) 142-152. https://doi.org/10.1016/j.phpro.2012.03.019

[10] H. Sato, T. Kamiyama and Y. Kiyanagi, Mater. Trans. 52 (2011) 1294-1302. https://doi.org/10.2320/matertrans.M2010328 
[11] H. Sato, T. Sato, Y. Shiota, T. Kamiyama, A. S. Tremsin, M. Ohnuma and Y. Kiyanagi, Mater. Trans. 56 (2015) 1147-1152. https://doi.org/10.2320/matertrans.M2015049

[12] H. Sato, K. Watanabe, K. Kiyokawa, R. Kiyanagi, K. Y. Hara, T. Kamiyama, M. Furusaka, T. Shinohara and Y. Kiyanagi, Phys. Procedia 88 (2017) 322-330.

https://doi.org/10.1016/j.phpro.2017.06.044

[13] W. Kockelmann, G. Frei, E. H. Lehmann, P. Vontobel and J. R. Santisteban, Nucl. Instrum. Methods A 578 (2007) 421-434. https://doi.org/10.1016/j.nima.2007.05.207 